\title{
An Approach for Effective Image Retrievals Based on Semantic Tagging and Generalized Gaussian Mixture Model
}

\author{
Anuradha. Padala \\ Gitam University / Department of Computer Science \& Engineering, Visakhapatnam, 530045, India \\ Email: hrushi@gitam.edu \\ Srinivas. Yarramalle \\ Gitam University / Department of Information Technology, Visakhapatnam, 530045, India \\ Email: ysrinivasit@ rediffmail.com \\ Krishna Prasad. MHM \\ JNTUK / Department of Computer Science and Engineering, Kakinada, 533003, India \\ Email: krishnaprasad.mhm@gmail.com
}

\begin{abstract}
The present day users navigate more using electronic gadgets, interacting with social networking sites and retrieving the images of interest from the information groups or similar groups. Most of the retrievals techniques are not much effective due to the semantic gap. Many models have been discussed for effective retrievals of the images based on feature extraction, label based and semantic rules. However effective retrievals of images are still a challenging task, model based techniques together with semantic attributes provide alternatives for efficient retrievals. This article is developed with the concepts of Generalized Gaussian Mixture Models and Semantic attributes. Flicker dataset is considered to experiment the model and efficiency is measured using Precision and Recall.
\end{abstract}

Index Terms-Social Networking, Flicker Database, Image Retrieval, Feature Extraction, Generalised GMM.

\section{INTRODUCTION}

The present day users are mostly associated with electronic gadgets. Mammoth information is pooled and stored across the globe in different formats for different usage. Tracing the relevant images from miniature collection is absolutely feasible by browsing; on the other hand, if the size of the database is massive containing diversified image collection, retrieval process becomes a challenging issue Also, as the databases increase in size, voluminous storage areas will be required. Today, with the advent of Social Networking, similar interest users are associated forming social networking groups and creating information hubs. As the number of users retrieving the information from the social media is increasing, effective techniques are to be evolved for extraction of relevant images. The majority of research in this area is projected using methods of tagging, relevance feedback, correlation, and image matching [1], [2]. Effective image retrievals are not possible, even after the digitization process, it is therefore necessary to use some effective mechanisms such as indexing, tagging etc, which help towards better management of the images. Classification techniques based on SVM, LDA, ANN are therefore considered [4], [5], [6] along with clustering algorithms based on EM, GMM and Monte-Carlo distribution, to address the issue [14]. In most of these models, feature extraction is done using either low level features, visual features (or) EXIF features. The features extracted are considered and a Semantic Relevance is formulated for retrieving the relevant image based on the query. However, this is totally a non-degenerative approach. But, Generative models based on mixture models will be very effective [S.K.Pal, N.R.Pal, (1993)] (Srinivas et al. (2007)) [8], [9], [10]. Hence, in this manuscript a model based approach is considered. The methodology for any image retrieval system for effective retrieval system ought to formulate a knowledge-base (PERNER, 1994). This knowledge-base should mainly consist of a brain storming module which helps to identify the problem domain for extracting important image particulars. In addition it consists of image description that aid to understand the various attributes of the images later assist to identify the features. In this paper, the relevant images are pooled together using the K-Means clustering algorithm. The knowledge base is built by extracting the Probability Densities of the image pixels, and Semantic Tags of the images are fused. The reset of the article is organized as follows. In section 2 of the paper the Generalized Gaussian Mixture Model (GGMM) is proposed. The feature extraction methodology is present in section 3 of the paper. The methodology is explained using a algorithm in section 4 , the section 5 of the paper presents the database considered; section- 6 of the paper presents the concept of K-Means algorithm, Section -7 of the paper deals with 
Relevant Scores, methodology and data base considered for experimentation in section 8 . The final section-9 concludes the paper.

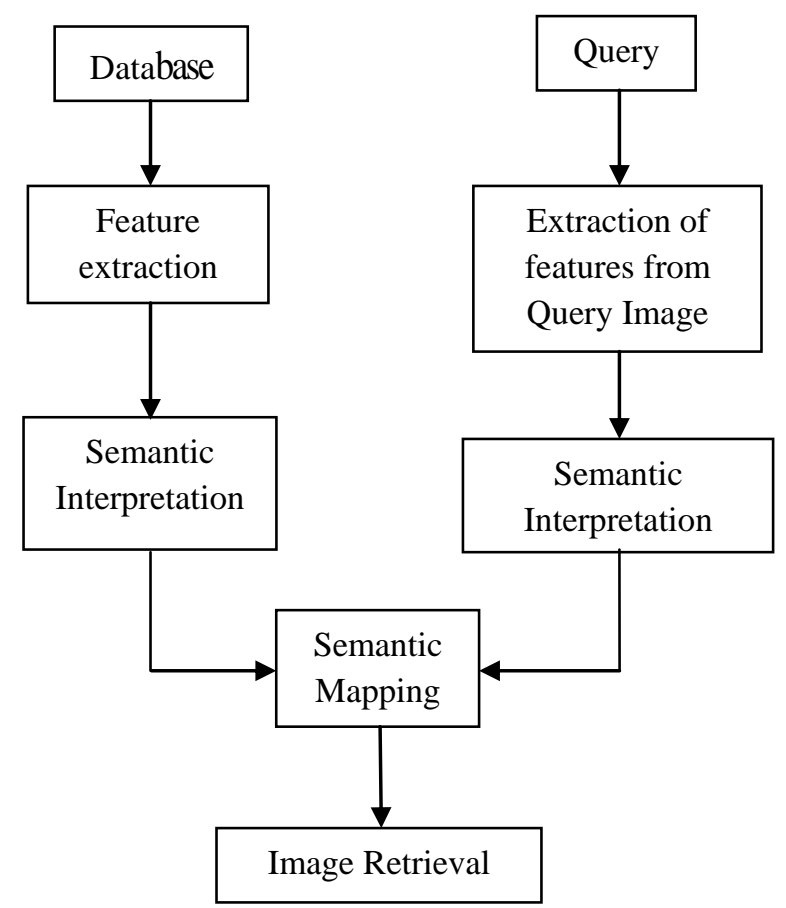

Fig 1: General Framework of Semantic Annotation based Retrieval

\section{Generalized Gaussian MiXture Model}

In this paper Generalized Gaussian Mixture Model is considered for the classification of the data. The choice of Generalized Gaussian Mixture Model is to classify the images more appropriative basing on the symmetry of distribution. It can handle images which are either leptokurtic or platy-kurtic [7]. The probability density function of the Generalized Gaussian Mixture Model is presented in the following equation.

$$
\begin{gathered}
f(z \mid \mu, \sigma, P)=\frac{1}{2 \Gamma\left(1+\frac{1}{P}\right) A(P, \sigma)} e^{-\left|\frac{\left(Z_{i}-\mu_{i}\right)}{A(P, \sigma)}\right|^{P}} \\
\sigma>0, A(P, \sigma)=\left[\frac{\sigma^{2} \Gamma(1 / P)}{\Gamma(3 / P)}\right]^{\frac{1}{2}}
\end{gathered}
$$

\section{FEATURE EXTRACTION}

The breed of images available in the internet increased exponentially, thereby retrieving meaningful images is becoming next to impossible. This has set a motivation to the researchers to consider the importance of Feature Extraction in retrieving the images from the database, and forced the researchers to track effective algorithms using feature extraction. Most of the information, which is generally retrieved by the users, can be in the following forms

- Textual articles in the form of images, information passage or human emotions, which are generally difficult to express in terms of words.

- Medical data which can be useful for analysis

- Data pertaining to bridges, monuments and other designs which can be used in later stages.

To retrieve the relevant data from the databases, user's make use of different types of features or attributes, which include

- blend of a particular colour, texture or contour features

- specific category of entity

- type of occurrence

- locations, or actions

- personal emotions);

- Metadata

Most of the historical reviews [5] in this area are based on text, where the descriptions regarding the images are explained and highlighted using textual information. However, with the abundant raise in the size as well as the scale of image databases, this approach becomes tedious [6]. To overcome this drawback, the low level features are considered for feature extraction. Extensive research in this direction has been carried out, in searching images of relevancy from the databases of Art galleries, Museums, Forecasting, Medical imaging, Forensics etc, but this mechanism of retrieval failed on bulky databases. The concepts of classifiers associated together with relevance feedback mechanisms gained popularity of late.

Image retrievals can be addressed broadly using

i) Primitive features, such as shape, location, texture and colour

ii) Middle level features, such as Query by Example mechanism.

iii) High-level features or Conceptual features.

Generally, the process of Feature extraction is carried out in the background, hence for efficient and effective retrievals, these features are to be associated with semantic interpretations. The semantic interpretations help to extract the data using the semantic attribute and also minimize the semantic gap. These semantic attributes are easily understood by the users when compared to the low level features which include contrast, symmetry, homogeneity and uniformity.

To reduce the search space, the dimensionality of the data is reduced by clustering the data, such that the homogenous images are together. In the present methodology, the images from the dataset are clustered using K-Means algorithm. The parameters from each of 
the images are extracted using the GGMM. Each of the images are Tagged and indexed. The Tags and the Probability Density Feature values are fused and these fused values are considered to be the features. These images are considered and the relevant image is retrieved based on the query using the relevant scores.

\section{IMAGE RETRIEVAL ALGORITHM}

Step 1. Consider the Image Dataset from Flicker, preprocess the images in the dataset to overcome noise

Step 2. For each input image, obtain the Probability Density Function (PDF) of the GGMM, by using equation 1 , of section II

Step 3. Associate a semantic Tag for each of the images of the dataset

Step 4. Fuse these two features using score level fusion

Step 5. Consider the Query image, pre-process the Image.

Step 6. Associate the Semantic Tag to the Query image

Step 7. Obtain the PDF of the Query image using GGMM

Step 8. Fuse these two features using score level fusion

Step 9. Map the PDF of the Query image against the PDF of the images in the Database and also map the semantic tags

Step 10. If match found, retrieve the image.

\section{DATABASE CONSIDERED}

In order to perform the experimentation we have considered the Flicker database consisting of 25,000 images with tags like nature, cigarette flowers and watches etc. Each image is associated with a tag description; among these images 450 have unique tags. The experimentation is performed by considering 100 images, Query image is considered with the size $100 \mathrm{x}$ 100 .

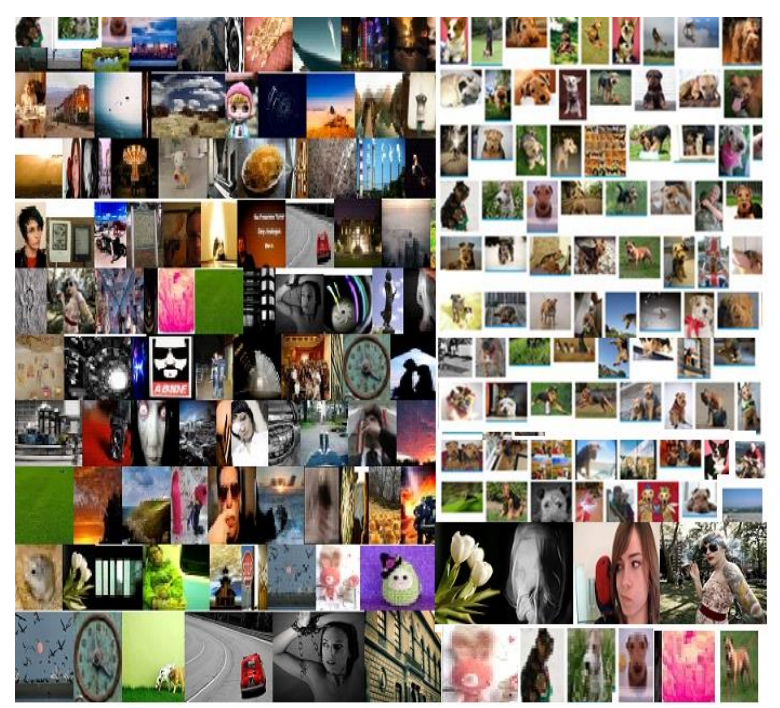

Fig 2: Database Considered from Flicker

\section{K-MEANS ALGORITHM}

In order to segment the image data, into homogenous groups, K-Means clustering is utilized in this paper. However, the main disadvantage of K-Means algorithm is the choice of $\mathrm{K}$ itself $[1,3]$. The histogram technique is used to overcome the drawback, the histograms of the images are considered and based on the peaks the initial value of $\mathrm{k}$ is assumed and the K-Means algorithm is performed. The stepwise procedure of the K-Means algorithm is presented below.

Step 1. Initiate with the primary $\mathrm{k}$. value

Step 2. From Step 1, we get the number of arbitrary clusters.

Step 3. Compute the centroid and assign each pixel into this cluster by assigning each pixel xi to the closest centroid, using Euclidian distance measure.

Step 4. Calculate the cluster assignment metrics using

$$
\sum_{i=1}^{k} u_{i j}=1 \forall i \& j
$$

where $\mathrm{u}$ is the cluster assignment matrix.

Step 5. If the cluster centroid or assigned matrix changes, repeat the above steps.

\section{RELEVANT SCORES}

The images from the clustered database are considered and the most relevant images are retrieved as follows

Let $\mathrm{t} 1$ be the image in a database and $\mathrm{t} 2$ be the query image; the relevance is obtained by using the following equation

$R(t 1, t 2)=\exp \left(\frac{\max (\log f(t 1), \log f(t 2)-\log f(t 1, t 2))}{\log M-\min (\log f(t 1), \log f(t 2))}\right)$

Where,

$\mathrm{f}(\mathrm{t} 1)$ and $\mathrm{f}(\mathrm{t} 2)$ denotes the number of images annotated with $\mathrm{t} 1$ i.e. the number of images having the same semantic tag.

$\mathrm{f}(\mathrm{t} 1, \mathrm{t} 2)$ denotes the image having similar semantic tags.

$\mathrm{M}$ denotes the total number of images in the data base.

\section{A. Tags Considered}

For effective retrievals, images with the tags-, bird, green, red, golden, white, flower and gauge are considered. The set of images retrieved based on relevant tags, after applying the above equation, are presented in Fig 3 


\begin{tabular}{|c|c|}
\hline Images & Tags \\
\hline & $\begin{array}{l}\text { pink,moon,pelicans,seagulls,flock, birds,flying } \\
\text { beach,sanfelipe,méxico,canoneos30,canon702 } \\
\text { 00mmf281,addtofeed,bajacalifornia, }\end{array}$ \\
\hline & sonja,parrot,bird, \\
\hline
\end{tabular}

Tag: Grass
\begin{tabular}{|c|l|}
\hline Images & \multicolumn{1}{c|}{ Tags } \\
\hline & $\begin{array}{l}\text { fun,special,dogs,dalmatian,dalmata,golden,re } \\
\text { triever,nature,animals,yard,green,grass,brazil } \\
\text {,brasil,jack,johnson,music,upside,down, }\end{array}$ \\
\hline & minimal,zurich,green,grass,someone,winner, \\
\hline
\end{tabular}

Tag: Red

\begin{tabular}{|l|l|} 
350d,ef50mm18,flowers,black, white,dof,ex \\
Tlored,flora,
\end{tabular}

Tag: Mexico

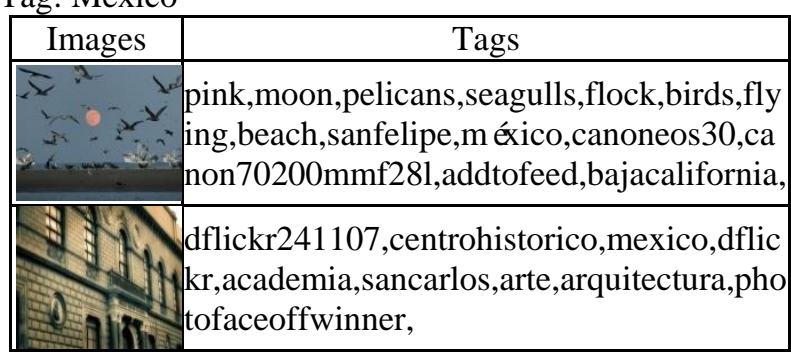

\begin{tabular}{|c|c|}
\hline Images & Tags \\
\hline & Cat, Grass, Green Lawn \\
\hline & Garden, Green, Dog \\
\hline
\end{tabular}

\begin{tabular}{|c|c|}
\hline Images & Tags \\
\hline Mag, standing, green floor, field \\
\hline
\end{tabular}

Tag: Flying

\begin{tabular}{|l|l|}
\hline Images & Tags \\
\hline & pink, moon,pelicans, seagulls, flock, birds,flying \\
& \\
& airplanes,airplane,flying,whileflying, \\
\hline
\end{tabular}

\begin{tabular}{|r||l|}
\hline \multicolumn{1}{|c|}{ Images } & \multicolumn{1}{|c|}{ Tags } \\
\hline$\ldots-$ & moon,,birds,flying,sky \\
\hline & Chair,red \\
\hline
\end{tabular}

Fig 3: Images with Tags

\section{EXPERIMENTATION}

From the above images with tags, the retrieval of images is not effective as seen in case of Flying objects, where the user interest may be a bird, but from the retrieved images with tag, it could be seen that Aircrafts and a lady are also retrieved. In case of retrieving the images with tag 'red', various other images where 'ed' consist as a suffix, are also retrieved. This can be seen under the tag 'red'. Hence tagging alone cannot retrieve the images effectively. Hence in this paper the retrieved tags are fused with the PDF of the Generalized Gaussian

Tag: Green 
Mixture Model for effective image retrievals.

\section{B. Score Level Fusion}

For efficient image retrievals, in this paper score level fusion is used, the procedure operates on a Logical AND/ OR operation, where the relevancy is indicated as ' $\mathrm{Y}$ ', and non-relevancy by ' $N$ '.

\section{Normalization}

Normalization is a process which is used to generate consistent values from the input images by overcoming the incompatibilities. The raw matching score be denoted using ' $\mathrm{s}$ ', and $\mathrm{S}$ denotes, the set of all images of relevancy, then the normalized score, given by ' $\mathrm{s}$, is given by as s, from the set $\mathrm{S}$ of all scores for that matcher, and the corresponding normalized score as s'.

$$
\text { Z-score: } s 1=(s-\mu) /(\sigma)
$$

where $\mu$ - denotes the mean of images and $\sigma$ specifies the standard deviation of the image data

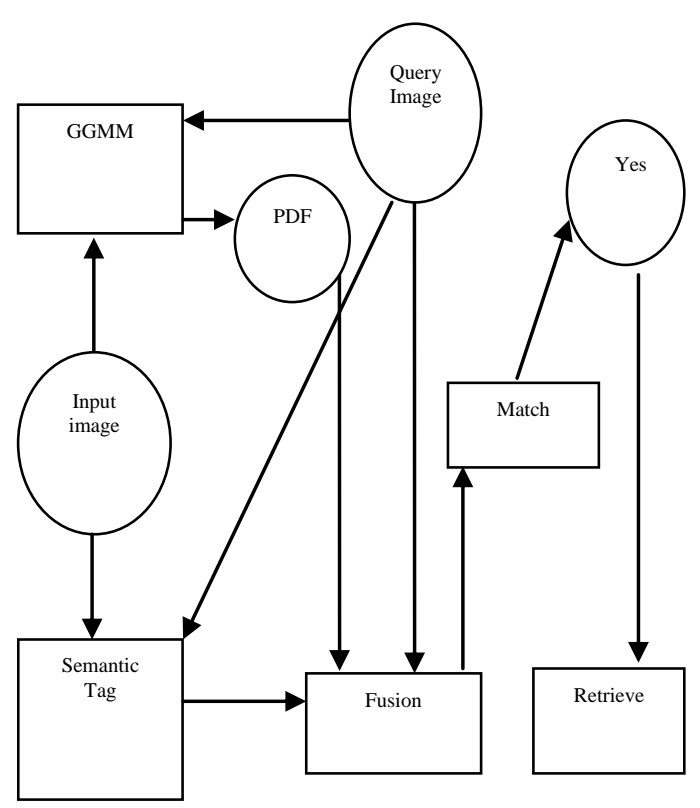

Fig 4: Image retrieval Process

In order to validate the model, the query image is given as input to find the relevancy among the images in the database. Each image in the database associated with a semantic tag is shown in Fig. 3.

Each image in the database is clustered using K-Means algorithm and for which the parameters are considered for the clustering process. The relevant images are grouped in different clusters. In order to reduce the search time, we have considered a relevance score approach where the relevance of the query image against the images in each of the cluster are computed and relevant images are extracted using the formula (2)

Searching becomes one of the key issues associated while retrieving relevant images, in particular using Content, from voluminous databases. Many metrics are used in the literature to measure the relativeness among retrieved and query images using, similarity based, ranking the images based on distance measures such as Manhattan, Euclidian, Cityblock distance, Chebychev, etc. In similarity based computations, the query image features and database image features are compared using the foresaid distance measures.

Metrics based on human perception are also mostly used for gauging the similarity between the images.

In order to evaluate the developed model we have considered the performance metrics based on human perception such as, precision and recall. The formula of which is given below.

\section{Precision}

It is the ratio of the numeral of related images retrieved to the total number of irrelevant and relevant images retrieved. It is generally expressed in percentage

Precision $=(\mathrm{A} /(\mathrm{A}+\mathrm{C}))^{*} 100$;

A: Number of relevant images retrieved.

C: Number of irrelevant images retrieved.

A + C: Total number of irrelevant + relevant images retrieved

\section{E. Recall}

It is the proportion of the number of appropriate images retrieved to the total number of relevant images in the database. It is frequently expressed in percentage.

Recall $=(\mathrm{A} /(\mathrm{A}+\mathrm{B})) * 100$

$A$ : Number of relevant images retrieved

B: Number of relevant images not retrieved

$\mathrm{A}+\mathrm{B}$ : The total number of relevant images

The efficiency of the developed model is evaluated by considering a test data of 200 images. The evaluation is carried out by generating test cases. We have performed experiments on the test database of 200 images. The results are given as follows

Table 1: Results of Precision and Recall Metrics

\begin{tabular}{|l|l|l|l|l|}
\hline $\begin{array}{l}\text { Total No of } \\
\text { Relevant } \\
\text { Images }\end{array}$ & $\begin{array}{l}\text { No of } \\
\text { Relevant } \\
\text { Images } \\
\text { Retrieved }\end{array}$ & $\begin{array}{l}\text { Total No of } \\
\text { Retrieved } \\
\text { images }\end{array}$ & Recall & Precision \\
\hline 35 & 22 & 33 & 54 & 62 \\
\hline 23 & 19 & 27 & 78.4 & 70.3 \\
\hline 19 & 16 & 22 & 66 & 80 \\
\hline 42 & 28 & 48 & 76 & 69 \\
\hline 39 & 30 & 42 & 71 & 65 \\
\hline
\end{tabular}

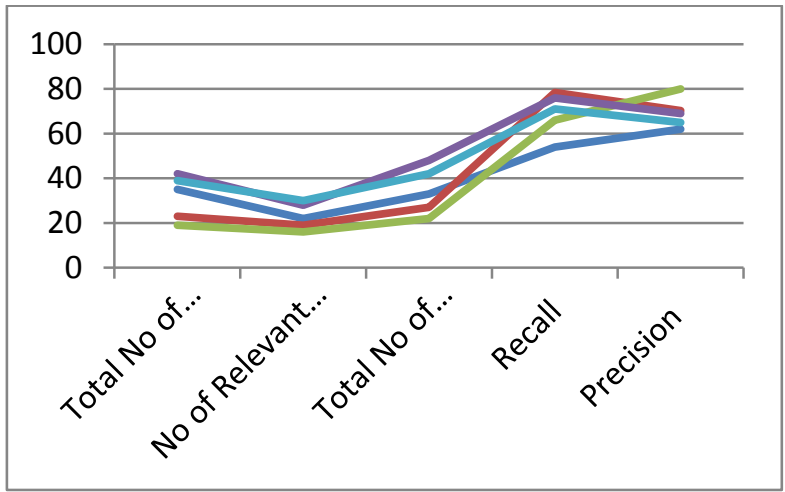

Fig 5: Graphical Representation of Precision and Recall values 


\section{CONCLUSION}

In this paper a novel approach for retrieving the query images from a social networking model is presented using Generalized Gaussian Mixture Model. In this article, the relevant images are retrieved from the database based on semantic tags associated with the PDF of the GGMM using Fusion. The developed methodology is evaluated using metrics like precision and recall. The results show that the developed model exhibits good retrieval accuracy.

\section{REFERENCES}

[1] Ramadass Sudhir, S. Santhosh Baboo, "A Efficient Content based Image Retrieval System using GMM and Relevance Feedback", International Journal of Computer Application, Vol. 72-Number 22,2013.

[2] Shanmugapriya, N. and R. Nallusamy, "A NEW CONTENT BASED IMAGE RETRIEVAL SYSTEM USING GMM AND RELEVANCE FEEDBACK", Journal of Computer Science, Vol.10, Issue-2, pp. 330340.

[3] Eskicioglu M.A and Fisher P.S (1995) "Image Quality Measures and their Performance", IEEE Transactions on Communications, Vol.43, No.12.

[4] Sumiti Bansal Er. Rishamjot Kaur, "A Review on Content Based Image Retrieval using SVM", International Journal of Advanced Research in Computer Science and Software Engineering, Vol.4, Issue-7, pp. 232-235, July 2014.

[5] Jia Li, "Linear Discriminant Analysis", URL: http://sites.stat.psu.edu/ jiali/course/stat597e/notes2/lda .pdf 10/09/2014 2:36 PM.

[6] D.Santhosh, Tina Esther Trueman, "Artificial Neural Network Technique for CBIR Based On Query Image Feature Extraction", International Journal of Innovative Research in Computer and Communication Engineering, Vol.2, Special Issue-3, July 2014.

[7] Sílvio M. Duarte Queirós, Nuno Crokidakis, and Diogo O. Soares-Pint, "Effect of platykurtic and leptokurtic distributions in the random-field Ising model: Meanfield approach", Phys. Rev. E 80, 011143 - Published 30 July 2009.

[8] Nikhil R Pal and Sankar K Pal, "A Review On Image Segmentation Techniques", Pattern Recognition, Vol. 26, Issue.9, pp 1277-1294, 1993.

[9] Y. Srinivas, “An Efficient Approach for Medical Image Segmentation Based on Truncated Skew Gaussian Mixture Model using K-Means Algorithm", International Journal of Computer Science and Telecommunications, Vol. 2, Issue 6, pp 81-88, Sep 2011.

[10] Yarramalle, Srinivas; Rao, K. Srinivas, "Unsupervised image segmentation using finite doubly truncated Gaussian mixture model and hierarchical clustering", Journal of Current Science, Vol. 93, Issue. 4, pp 507, 2007.

[11] S.Najimun Nisha, Mrs.K.A.Mehar Ban, "An Enhanced Image Retrieval Using K-Mean Clustering Algorithm in Integrating Text and Visual Features", International Journal of Innovative Science, Engineering \& Technology, Vol. 1 Issue 1,pp 10-15, March 2014.

[12] Cheng, "Color Image Segmentation:Advances and Prospects", Pattern Recognition, Vo1.34, pp2259-2281, 2001.
[13] D. Martin, C. Fowlkes,D. Tal, and J.Malik "A database of human Segmented natural images and its application to evaluating segmentation algorithms and measuring ecological statistics", in proceedings of 8th International Conference on Computervision, vol.2, pp.416-423, 2006.

[14] T. Yamazaki, Tatsuya Yamazaki, "Introduction of EM Algorithm into Color Image Segmentation”, CiteSeer, Venue: Proc. ICIPS'98.

[15] Jeff A.Bilmes, "A Gentle Tutorial of the EM Algorithm and its application to Parameter Estimation for Gaussian Mixture and Hidden Markov Models", Technical Report, University of Berkeley, ICSI-TR-97021, 1997.

[16] Lie. T, Sewehand.W, "Statistical approach to X-ray CT imaging and its applications in image analysis", IEEE Trans. Med. Image. Vol.11, No.1, pp 53-61, 1992.

[17] Kelly. P.A, "Adaptive segmentation of speckled images using a hierarchical random field model". IEEE Transactions Acoust. Speech. Signal Processing, Vol.36, No.10, pp.1628-1641, 1988.

[18] Mclanchlan G, Krishnan T, "The EM Algorithm and Extensions", John Wiley and Sons, New York -1997.

[19] Mclanchlan G, Peel.D, "The EM Algorithm For Parameter Estimations", John Wiley and Sons, New York -2000 .

[20] Vishal Jain, Dr. Mayank Singh, "Ontology Based Information Retrieval in Schematic Web: A Survey", I.J. Information Technology and Computer Science, No.10, pp 62-69, 2013.

[21] Hadi A. Alnabriss, Ibrahim S.I. Abuhaiba, "Improved Image Retrieval With Color And Angle Representation", I.J. Information Technology and Computer Science, No.06, pp 68-81, 2014.

\section{Author's Profile}

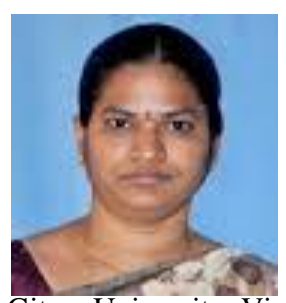

Anuradha. Padala has completed her M.Tech Degree in Computer Science and Engineering, Andhra University, Visakhapatnam. Her research area includes Image Retrieval, Semantic Web. Currently she is working as Assistant Professor in the Department of Computer Science Engineering, Gitam University, Visakhapatnam.

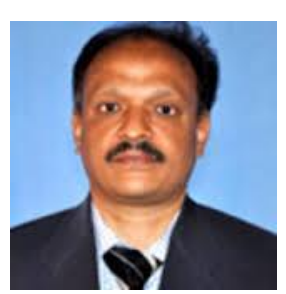

Srinivas $\mathbf{Y}$ is presently working as a Professor, in Department of Information Technology, Gitam University, Visakhapatnam. His research area includes Image Processing, Data Mining, and Software Engineering. He also Guided 6 PhD's.

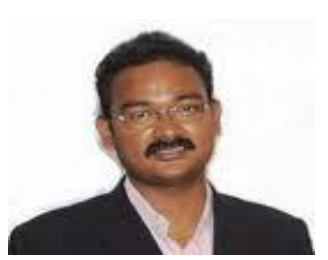

Krishna Prasad M.H.M is presently working as an Associate Professor in JNTU Kakinada in the Department of Computer Science Engineering. His research areas include Data Mining, Software Engineering. 